\title{
Comportamento di una grande diga sotto il gelo spinto
}

\author{
P. CaLoi $\left({ }^{*}\right)-$ M. Migani $(* *)-$ M. C. Spade $\left({ }^{*}\right)$
}

Ricevuto il 25 Maggio 1972

Riassunto. - In sintesi, il giuoco delle spinte, a cui è soggetta la diga di Pieve di Cadore durante l'anno, a parità di altre condizioni, può essenzialmente essere diviso in tre periodi.

1. - Verso la fine di Giugno, la temperatura dell'aria supera, in media, quella dell'acqua nel bacino a monte della diga: questa flette verso monte, con curvatura crescente dalla base al coronamento.

2. - Verso la fine di Ottobre, la situazione termica si inverte: la media temperatura dell'aria diviene inferiore alla media temperatura dell'acqua: la diga comincia a flettere verso valle.

3. - Quando la temperatura dell'aria è nettamente sotto lo zero, il giuoco delle spinte si fa più complesso: il sistema roccioso a valle della diga (imbevuto d'acqua), gelando si gonfia e spinge la base della diga verso monte; in quota, continua invece la spinta verso valle.

$\grave{E}$ in questa fase che, vinta la fermezza del nezzo, il contrasto fra roccia e manufatto può sfociare in minutissime fratture, rivelate - sotto forma di microscosse (energia $\sim 10^{10}-10^{11}$ e g) - dalla stazione sismica, funzionante a quota $660 \mathrm{~m}$ del concio centrale della diga.

1. - I noto che, al di sopra di $0^{\circ} \mathrm{C}$, il comportamento di una diga sotto l'azione delle variazioni di temperatura, si può sintetizzare in due principali movimenti: piegamento verso monte, per temperatura in aumento, flessione verso valle per temperatura in diminuzione ('). Questo, naturalmente, a prescindere dalle variazioni legate all'invaso.

$\left.{ }^{*}\right)$ Istituto Nazionale di Geofisica, Roma.

(**) E.N.E.L. Compartimento di Venezia. 
All'intorno dello zero e sotto lo zero, il comportamento di una diga si fa più complesso.

A monte dello sbarramento, il lago copre la diga fino arl una certa quota. Quando la temperatura scende sotto lo zero, lo strato d'acqua superficiale gela, per uno spessore più o meno grande, a seconda del salto termico negativo. Al di sotto dello strato gelato, l'acqua conserva una temperatura superiore a $0^{\circ} \mathrm{C}$. A valle, la situazione è quella legata alla temperatura esterna, cioè più o meno nettamente sotto lo zero. In queste condizioni, la parte di diga coperta dall'acqua, viene a trovarsi a temperatura superiore di quella a cui si trova la riga a. valle: di qui, almeno inizialmente, un'accentuazione della spinta del manufatto verso valle.

Ma se la temperatura dell'aria persiste a lungo, e nettamente, sotto lo zero, intervengono fenomeni nuovi, legati all'anomalo comportamento del suolo ghiacciato. Il sistema roccioso su cui poggia la diga è ovviamente imbevuto d'acqua, fino ad una certa profondità. Quando la temperatura si conserva sensibilmente sotto lo zero per parecchi giorni, il gelo penetra, più o meno profonclamente, nel sottosuolo a valle della diga. Gelando, la componente liquida provoca un rigonfiamento, tanto più sensibile, quanto più a fondo penetra il gelo. Ai margini del piazzale, a contatto con la diga, si manifesta pertanto una spinta verso lo sbarramento che - superata la spinta opposta dalla parte della superficie sommersa - finisce col tradursi in una flessione verso monte della base del manufatto. In quota, infatti, lo sbarramento subisce l'azione di rigonfiamento del mezzo roccioso d'imposta, solo nella ristretta zona, corrispondente allo spessore della diga; azione evidentemente inefficace ai fini di una spinta in direzione valle-monte; sicché, al di sopra del livello del piazzale esterno alla. diga si fa via via prevalente la spinta verso valle, come è schematizzato nella fig. 1 .

Che le cose procedano in questo modo, è testimoniato dalle registrazioni clinografiche, che si ottengono - nelle predette condizioni - a quote diverse del concio centrale della diga di Pieve di Cadore; e precisamente a quota piazzale (Pian delle Ere, quota 625m), a quota $660 \mathrm{~m}$ e a quota coronamento $(682 \mathrm{~m})$, fig. 2.

2. - Fin qui, la roccia e il calcestruzzo hanno risposto alle sollecitazioni contrastanti nei limiti della loro fermezza; ma quando il gelo è spinto nel tempo (e, quindi, nello spazio), cresce l'intensità della forza di spinta della roccia d'imposta, a valle della diga, verso lo sbar- 
Fig. 1 -- Rappresentazione schematica delle spinte cui i soggetta la diga di Pieve di Cadore, in tre diversi periodi dell'anno:

1. - Verso la fine di Giugno la temperatura dellaria supera, in media, quella dell acqua nel bacino a monte della diga: questa flette verso monte, con curvatura rescente dalla base al coronamento.

2. - Verso la fine di ot. tobre, la situazione termica s inverte qquella rappresentata in figura, rappresenta $u n$ momento di tale inversione specie per quanto riguarda il lago, dove $\dot{c}$ in atto un rimescolamento continuo dellacque): la diga flette verso valle.

3. - Quando la tempe. ratura dell'aria è nettamente sotto lo zero, il gioco delle spinte si fa più complesso: il sistema roccioso a valle della diga (imbevuto d'acqua) gelando si gonfia e spinge la base della diga verso monte; in quota continua, invece, la spinta verso valle. ì in que. sta fase che, vinta la fermezza del mezzo, il contrasto fra roccia e diga può sfociare in minutissime fratture $\left(10^{10}-10^{11}\right.$ erg), rivelate sotto forma di microscosse dalla stazione sismica funzionante a quota 660 del concio centrale della diga.

\section{Fine Giugno}
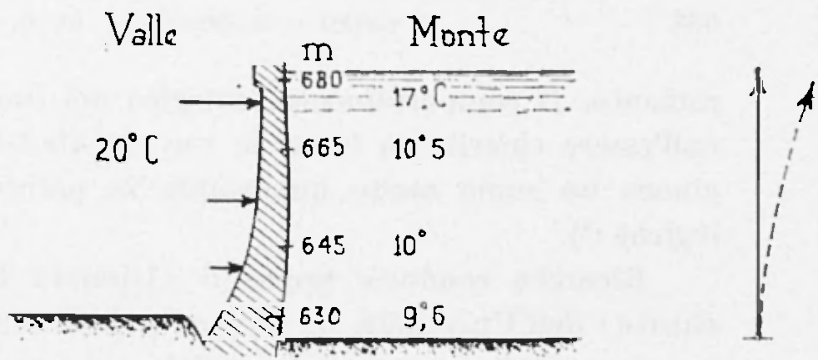

\section{Fine Otzobre}
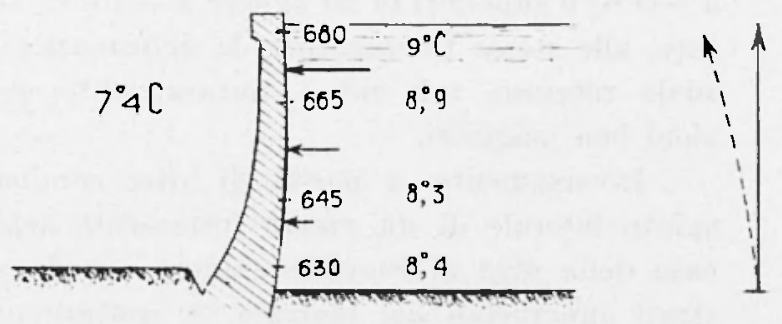

\section{Periadi invernali con gelo spinto}

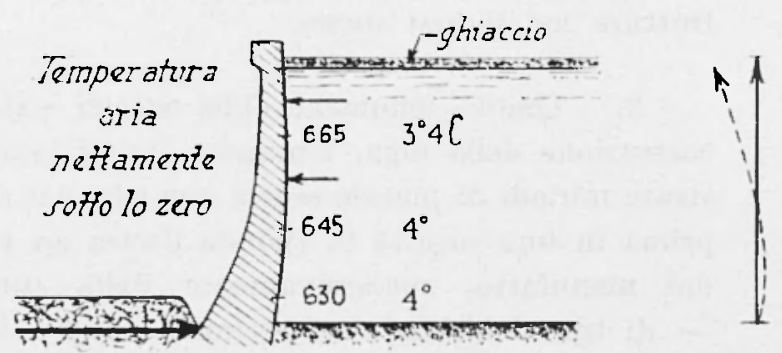

Fig. 1. - Conventional representation of the thrusts to which the dam of Pieve di Cadore is subiected yearly during three different periods:

1. - About the end of June. the air temperature, on the average, overcomes the water one in the watershed upstream the dam: the bending of the dam upstream increases from the bottom to the top.

2. - About the end of October, the thermal conditions change (the figure shows a moment of this inversion especially as to the Lake in the which a continuous blending of the water is happening): the dam bends downstream.

3 - When the air temperature is distincly below $0^{\circ} \mathrm{C}$ the action of the thrusts grows more complex: the rocky waterlogged system downstream of the dam, while is cooling, swells and pushes the dam bottom upstream; at the higher quote, on the contrary, the thrust downstream continues.

When the strenght limit of the medium is surpassed, a contrast between the rocky system and the concrete structure arises: this contrast can originate very small fractures revealed from seismic station installed into the central ashlar ( $0^{\circ} \mathrm{XIV}$ ) at 660 metres height of the dam, under the form of microshocks which energy is of about $10^{10} .10^{11} \mathrm{erg}$. 
ramento. Il comportamento reologico del suolo gelato è ancora lungi dall'essere chiarito in tutte le sue caratteristiche. In ogni caso, esso giuoca un ruolo molto importante in parecchie strutture geomorfologiche (i).

Ricerche condotte presso il "Disaster Prevention Research Institute " dell'Università di Kyoto $\left({ }^{8}\right)$, hanno provato - operando con esperimenti di compressione assiale su campioni di suolo ghiacciato estratti a diverse profondità, nella pianura di Tokyo - che i più elevati valori resi da sedimenti di argilla risultarono fra 10 e $20 \mathrm{~kg} / \mathrm{cm}^{2}$ a - 5 oc e superiori di $20 \mathrm{~kg} / \mathrm{cm}^{2}$ a $-10^{\circ} \mathrm{C}$. In caso di sabbie sedimentate, alle stesse temperature, la deformazione risulta più grande. Per suolo roccioso, tali valori, naturalmente, possono assumere proporzioni ben maggiori.

Inversamente, a parità di altre condizioni, aumenta quindi la spinta laterale di un mezzo fortemente gelato. Se poi - come nel caso della diga di Pieve di Cadore - alla spinta verso monte degli strati superficiali del piazzale, si contrappone la spinta verso valle della base della diga immersa nel lago, ciò crea un contrasto crescente, al crescere del gelo, fino al punto in cui, superato il limite di fermezza del mezzo interessato, si verificano rotture d'equilibrio, con conseguenti fratture nel mezzo stesso.

3. - Questo fenomeno ebbe aspetti vistosi nei primi anni dalla costruzione della diga. Durante i primi inverni, infatti, furono registrate miriadi di piccole scosse sismiche dai sismografi sistemati, dapprima in una casetta in sponda destra ad una cinquantina di metri dal manufatto: successivamente dalla stessa terna di sismografi - di tipo Ishimoto - sistemati, sempre in sponda destra, presso la cabina comandi a ridosso della diga $\left({ }^{2}\right)$ (sismografi più tardi sostituiti da una più potente terna di apparecchi "Girlanda" " La maggior parte di tali scossette proveniva dalla base dello sbarramento $(1,2)$.

Dopo alcuni anni, la stazione sismica funzionante negli scantinati della cabina comandi cessò di registrare microscosse provenienti dalla base dello sbarramento.

Caloi, che aveva seguito il fenomeno fin dal suo primo insorgere, era però convinto che esso non si fosse del tutto esaurito. Solo una stazione sismica, sistemata entro la diga, avrebbe potuto risolvere la questione. Ottenuto il permesso dall'" Enel ", una stazione di sismografi "Girlanda ", fu sistemata dal dott. Migani in un cunicolo a 
Al

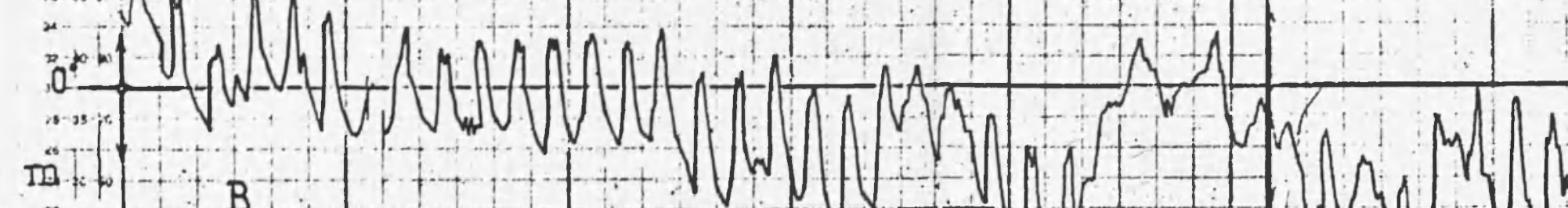
$m_{0}+1$
680

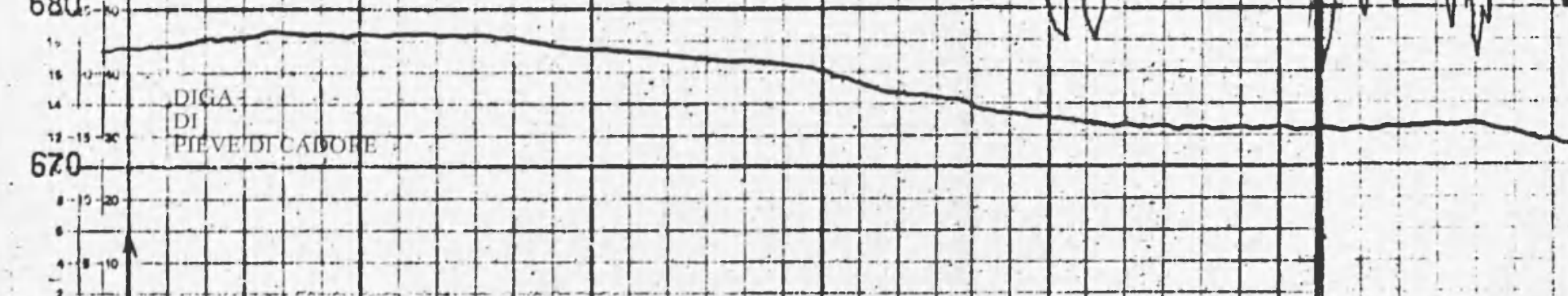
SŔ̃

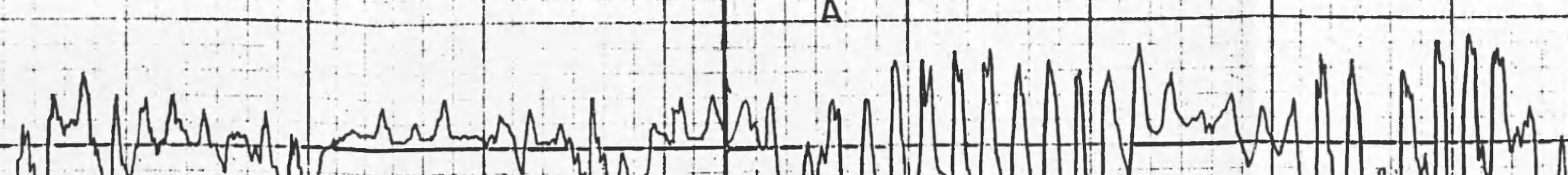

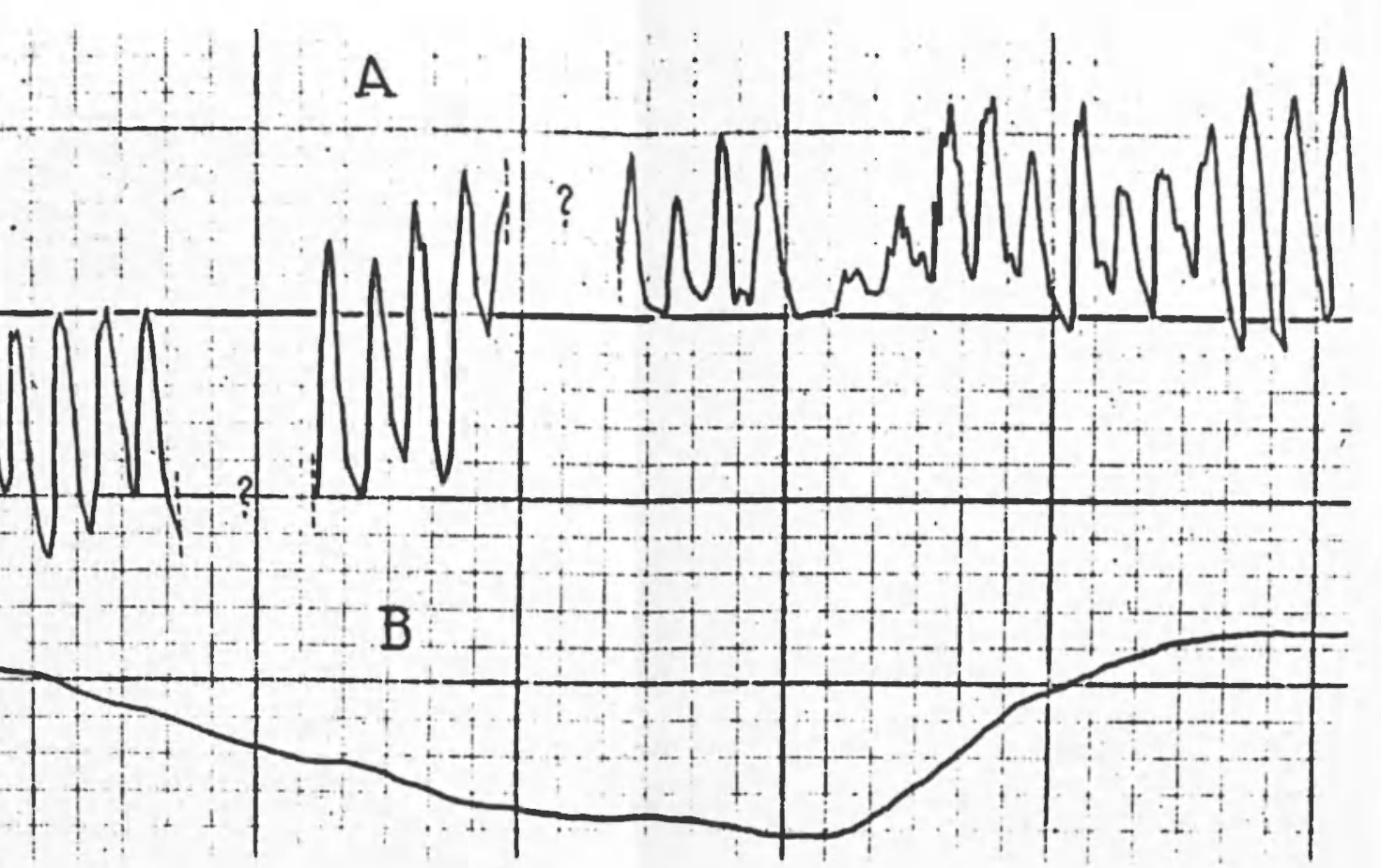
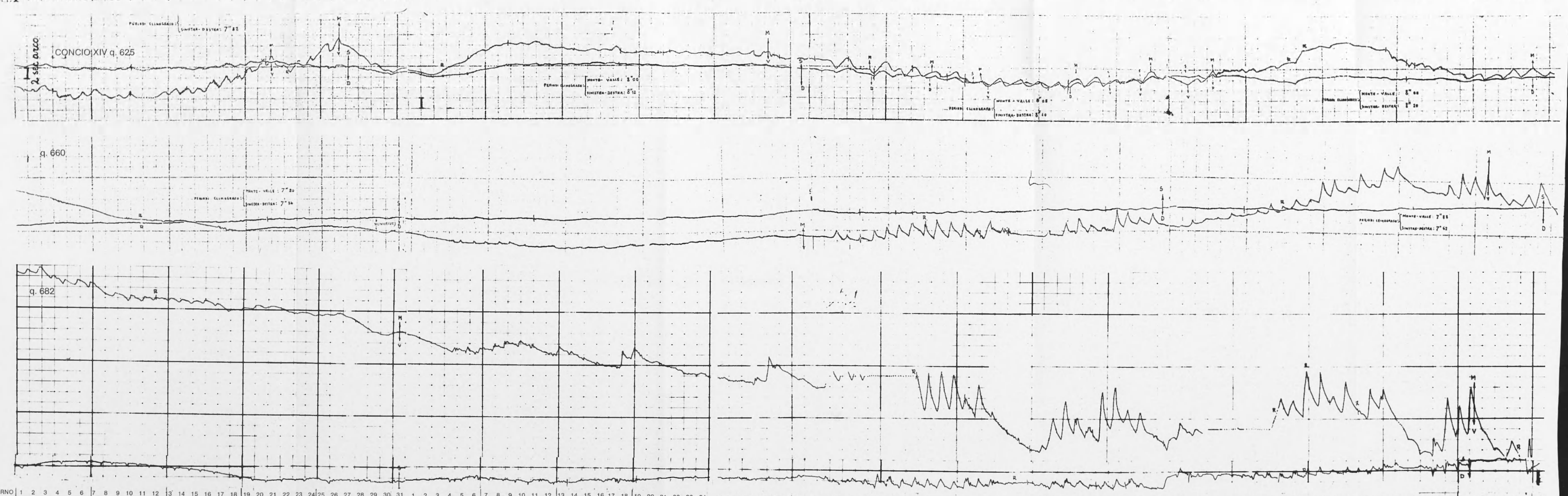

DICEMBRE 1970

GENNAIO 1971

FEBBRAIO 1971

MARZO 1971

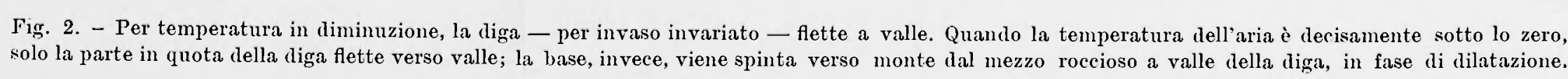

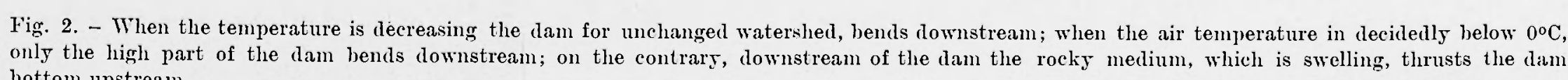


q. 660, nel concio XIV (centrale) della diga (Figg. 3, 4). Tale stazione, che ha permesso di rivelare nuovi fenomeni, di alcuni dei quali si ì trattato altrove e di altri si parlerà in seguito, ha consentito il rilevamento di centinaia di minutissime scosse, in giorni di gelo spinto; scosse che sfuggono all'analoga stazione funzionante in cabina comandi centralizzati (Figg. 5, 6).

A questo punto, viene spontaneo di chiedersi perché, nei primi tempi, le microscosse furono tanto più intense da essere registrate chiaramente - a migliaia - anche fuori della diga. La risposta i ovvia. Inizialmente, avevamo due mezzi - il sistema roccioso e la diga - con proprie caratteristiche, ben distinte. La loro forzata " convivenza" non poteva non portare a contrasti iniziali di una certa violenza, atti a smussare le più forti resistenze della roccia ai movimenti della diga: la fratturazione, pertanto, riuscì più generale ed interessò gli elementi di maggiori dimensioni: di qui la maggiore energia liberata dalle microscosse. Del resto, solo in questo modo il sistema poteva stabilirsi su nuove posizioni di equilibrio. [Non va dimenticato, a questo riguardo, che il modulo di elasticità del sistema roccioso sopportante il piazzale a valle della diga, in pochi anni - i primi - subi una fortissima flessione: dagli iniziali $7000 \mathrm{~m} / \mathrm{sec}$ della velocità delle onde longitudinali (1949), si passò ai $4500 \mathrm{~m} / \mathrm{sec}$ $\left.(1960)\left({ }^{4}\right)\right]$.

Superata la fase acuta della crisi, nella zona di contatto fra la culla dello sbarramento e il calcestruzzo, i micromovimenti di questo ultimo poterono realizzarsi in campo plastico. Non cosi durante i periodi di gelo spinto, nei quali, per i motivi sopra esposti, si verificano fratture, sorgenti di onde elastiche.

La fig. 6 dà numerosi esempi di microscosse, registrate dal Dicembre 1970 al Marzo 1971, nei periodi in cui la temperatura scese decisamente sotto lo zero $\left(^{*}\right)$. Noi, naturalmente, ne abbiamo

(*) A questo proposito, un osservazione superficiale potrebbe attribuire le microscosse alle crepe che - di quando in quando - si verificano nel ghiaccio del lago. A parte il fatto che queste si manifestano sporadicamente e, comunque, in numero limitato, va tenuto presente che - a motivo delle continue variazioni di livello nell'invaso - Io strato ghiacciato si presenta largamente fratturato in tutta una striscia torno torno alle sponde, alle quali quindi non aderisce rigidamente, disperdendo l'enezgia proveniente dalle spaccature, che pertanto perviene alla riva in misura minima. 


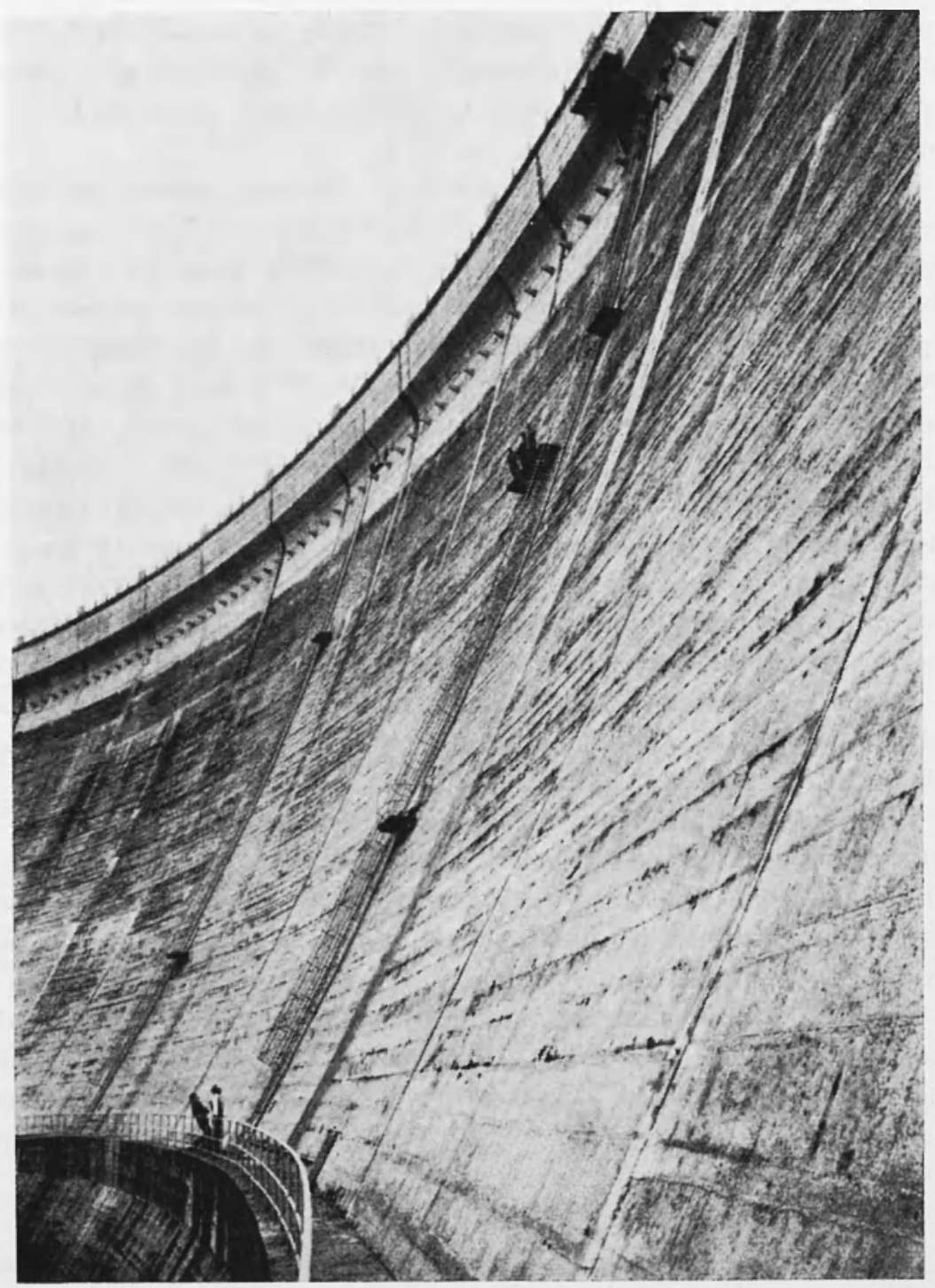

Fig. 3 - Diga di Pieve di Cadore. 


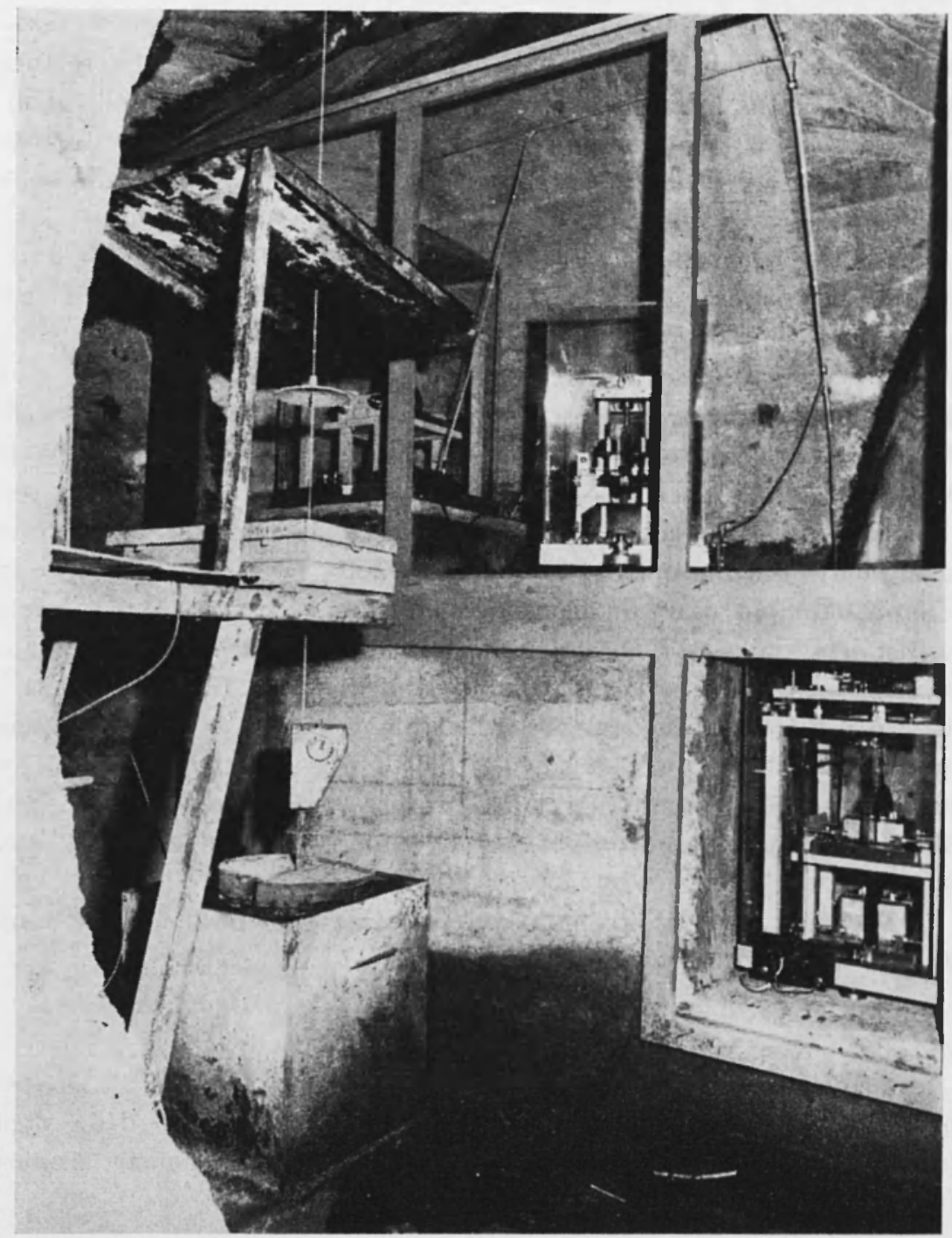

Fig. 4 - Terna di sismografi "Girlanda " installata dentro il concio ('entrale (XIV) della diga a quota $660 \mathrm{~m}$ (vedi fig. 3).

Fig. 4 - Tern of "Girlanda" seismographs installed and working into the central ashlar (XIV) at 660 ms height of the dam (see fig. 3 ). 
riportato solo alcune. (iia da esse, però, appare chiara la grande varietà di aspetti che presentano, e che le differenziano - talvolta notevolmente - l'una dall'altra. Ia differenziazione è testimonianza di diversa origine e di differente propagazione. La rottura d'equilibrio può avvenire lievemente discosta dalla base della diga. Jata la poca energia in giuoco, essa non sarà in grado di provocare lo scuotimento del manufatto che si limiterà a far da veicolo alle onde elastiche: avremo, in questi casi, oscillazioni rapidissime, che si esauriscono nell'intervallo di uno o due secondi (fig. 6). Se la frattura è più prossima alla diga, la sua energia può essere capace di provocarne lo scuotimento; trattandosi dell'eccitazione di elementi finiti, essi presentano analogie con il moto di un diapason: avremo cioè dispersione anomala, con oscillazioni di periodo via via più lungo, che saranno le più tarde e tenderanno ai valori propri dell'uninodale dei conci e - spesso - della diga stessa (5) (fig. 6). A questo riguardo, si noterà che le oscillazioni libere del manufatto, a volte insorgono immediatamente dopo l'inizio della perturbazione, a volte vi appaiono più o meno distanziate. Ciò sta a significare che varia la distanza, dalka stazione d'osservazione (nel caso specifico, sistemata in zona centrale della diga) della zona di disturbo. Va ancora sottolimeato il fatto che, sovente, la registrazione non ha le caratteristiche proprie di una microscossa singola, ma di più scotimenti, originati a breve intervallo di tempo l'uno dall'altro: è come se la rottura d'equilibrio in un punto, ne provocasse di analoghe in tutto un intorno (fig. 6).

Ia sugli aspetti predominanti delle registrazioni, ci riserviamo di ritornare in una prossima pubblicazione.

Segue un elenco di microscosse registrate il 24-25-26-27 Dicembre 1970 dalla stazione sismica funzionante nell'interno della diga (alcune di esse sono state registrate anche dalla stazione sismica funzionante negli scantinati della cabina comandi). 


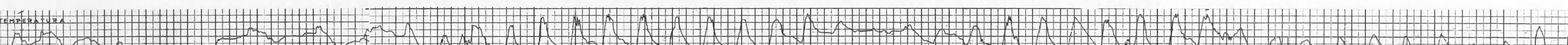

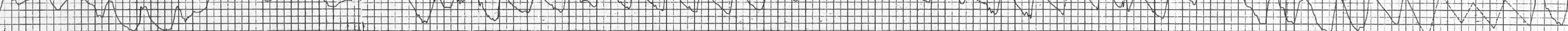




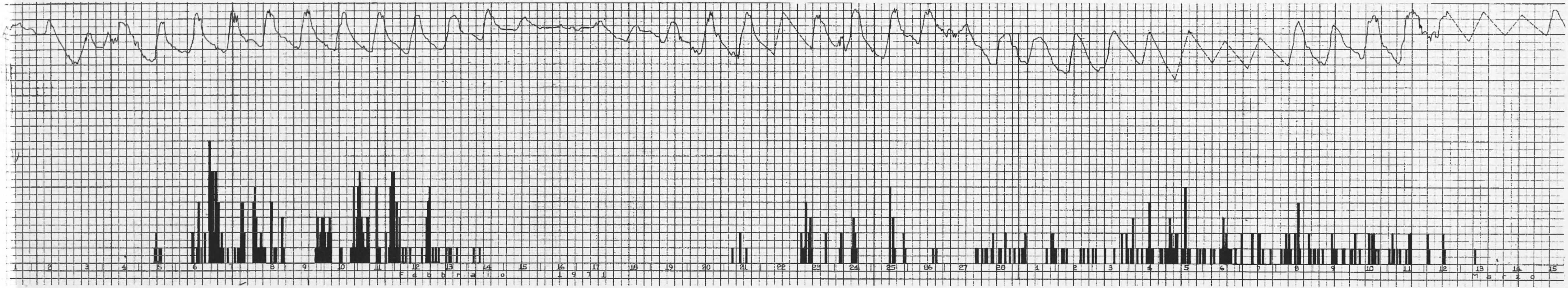

Fig. 5. - Andamento della temperatura dell aria e successione delle microscosse: queste ultime insorgono numerose quando la temperatura è decisa
mente

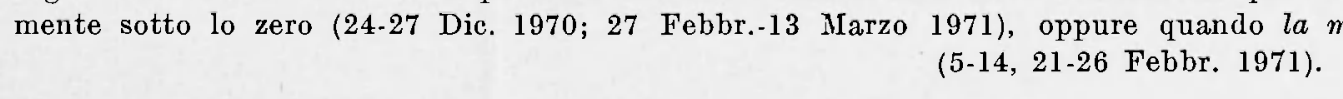

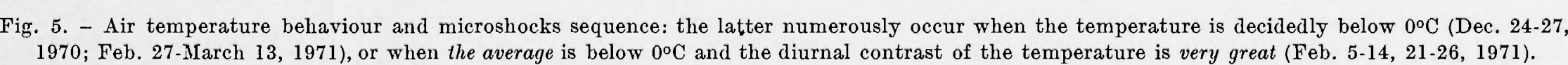




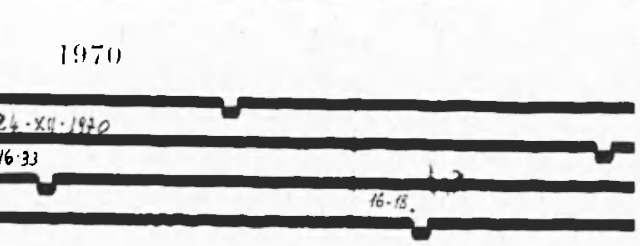

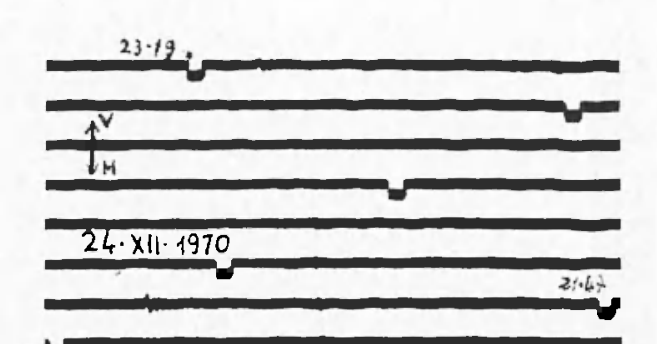

1971
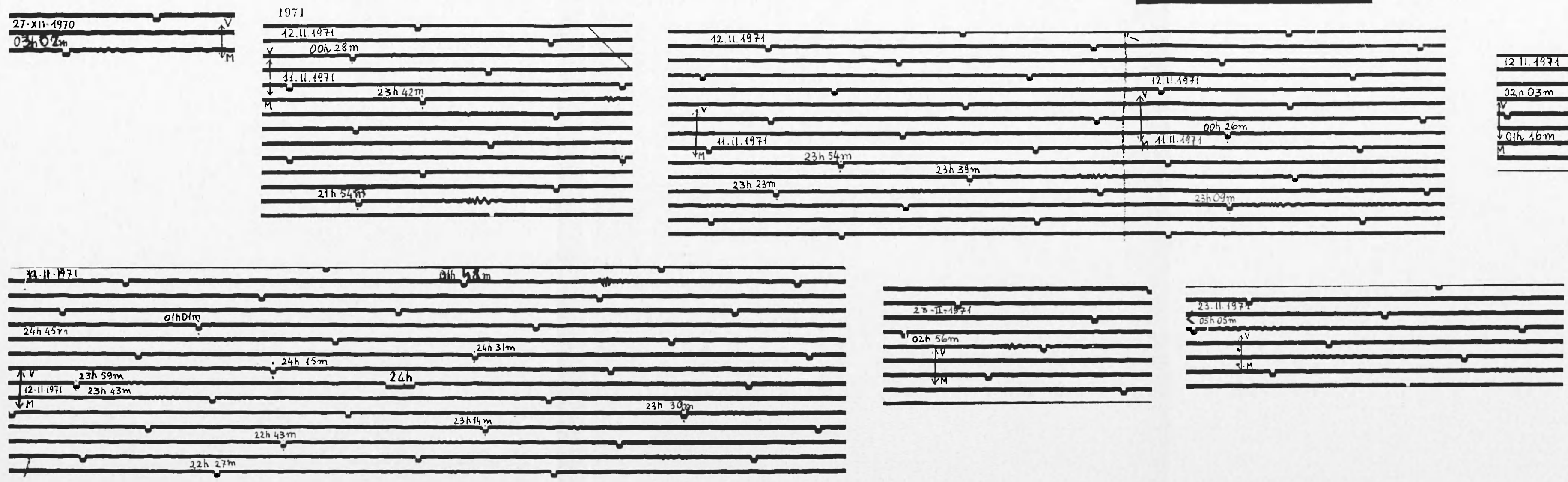

O4h $060 \mathrm{~m}$

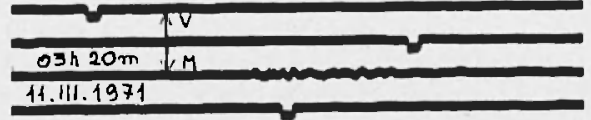

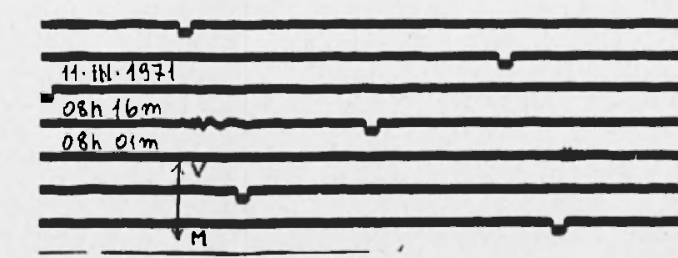

1211.197 .1 $02103 m$

tolk $16 \mathrm{~m}$ $\sqrt{21 / 22 \mathrm{~m}}$ $\frac{25 \times 101}{4-2}$ r. $s_{m}{ }^{2}$ taxy

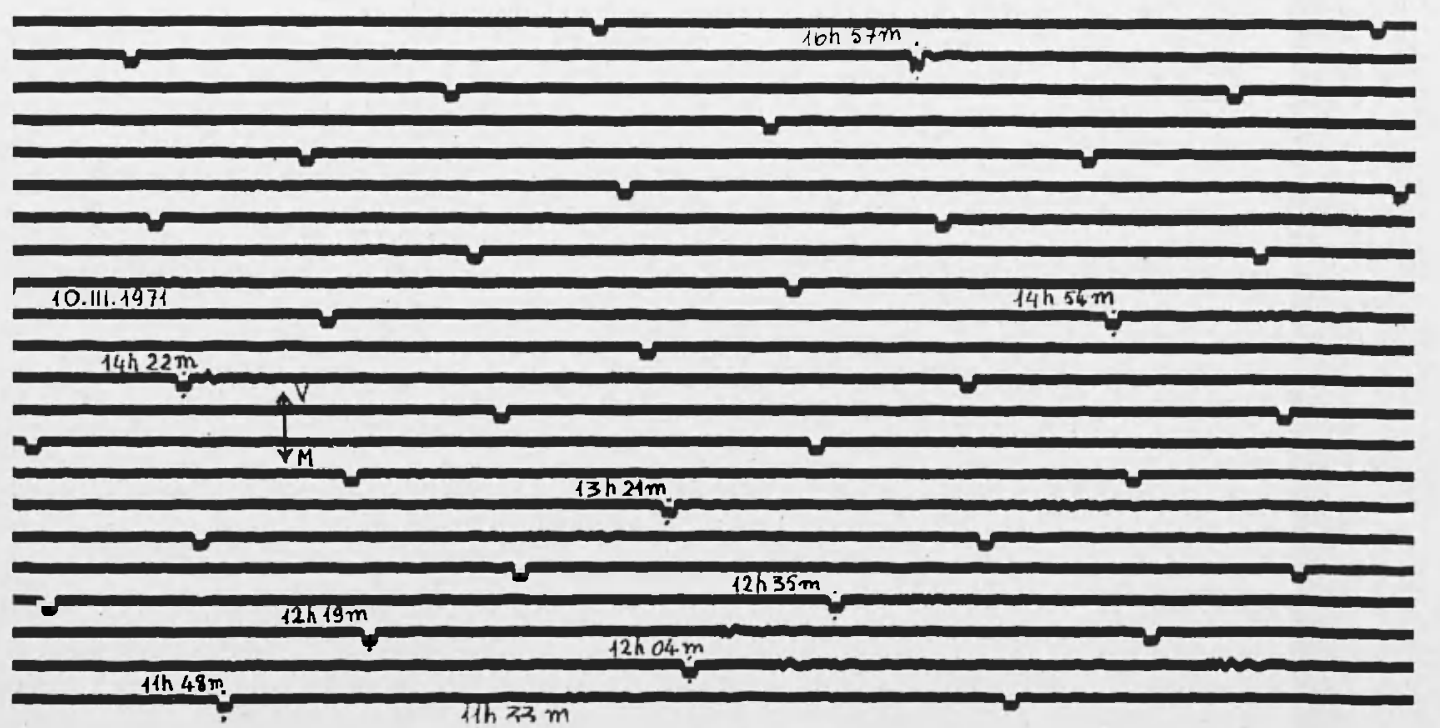

10.11.19:

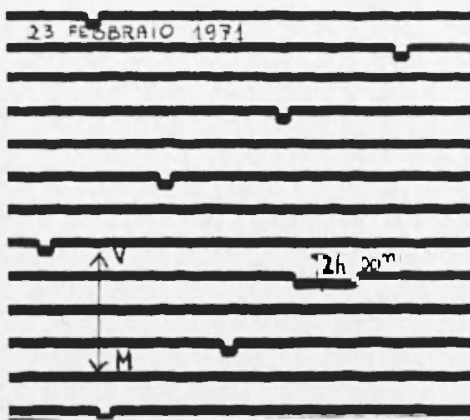

ish42m

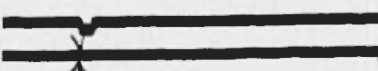

$+$

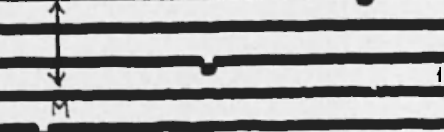




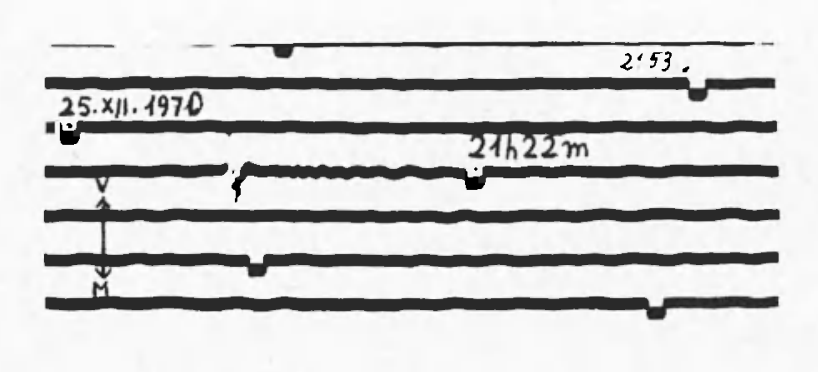

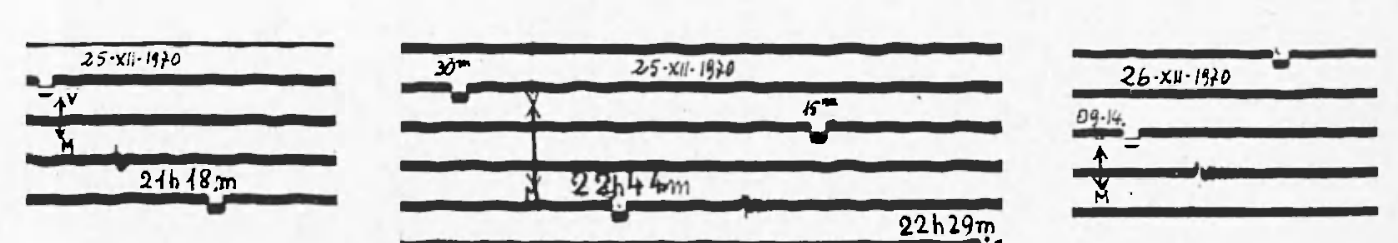

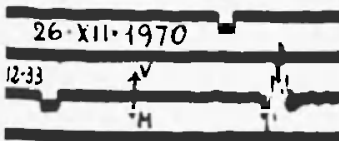

$\frac{16 \cdot \times 11 \cdot 1970-}{12 \cdot 33}+1$

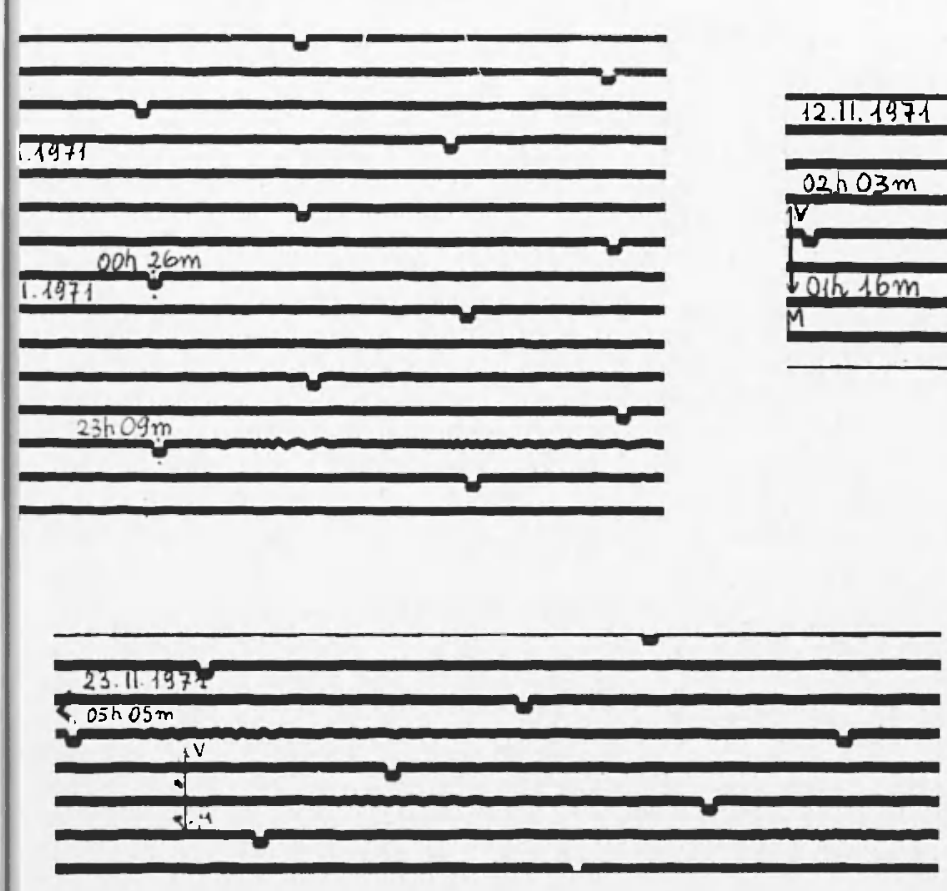

12.11.1971 02,03m Th1616m -

-

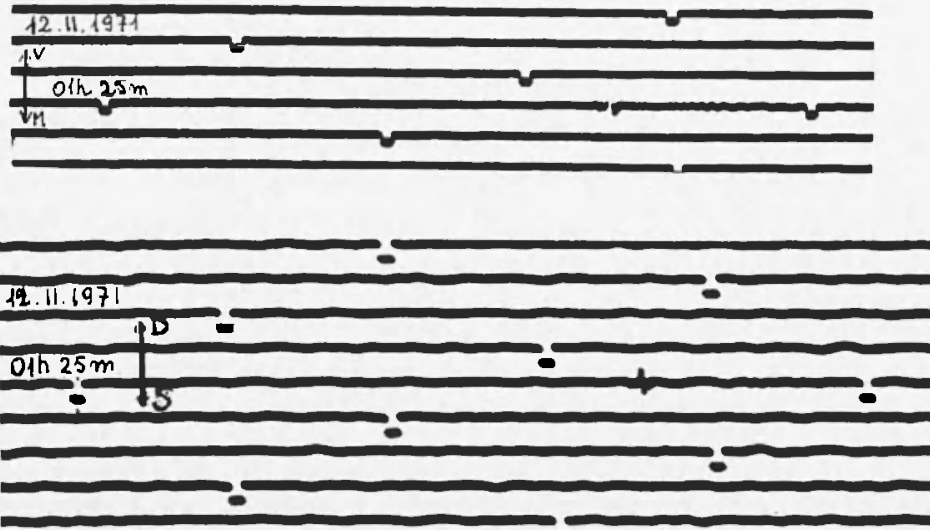

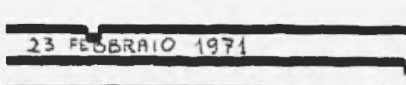
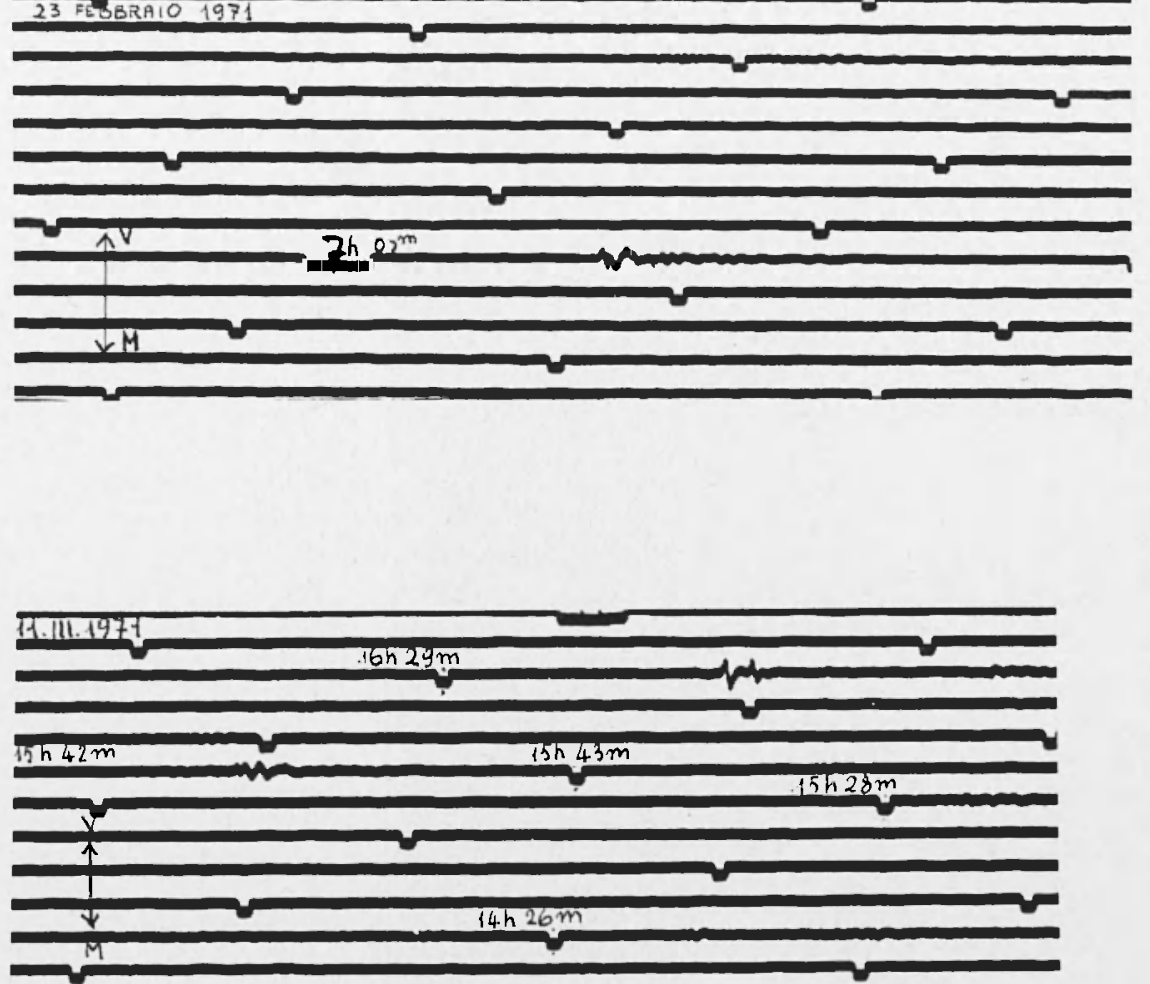

2.111.1997 -
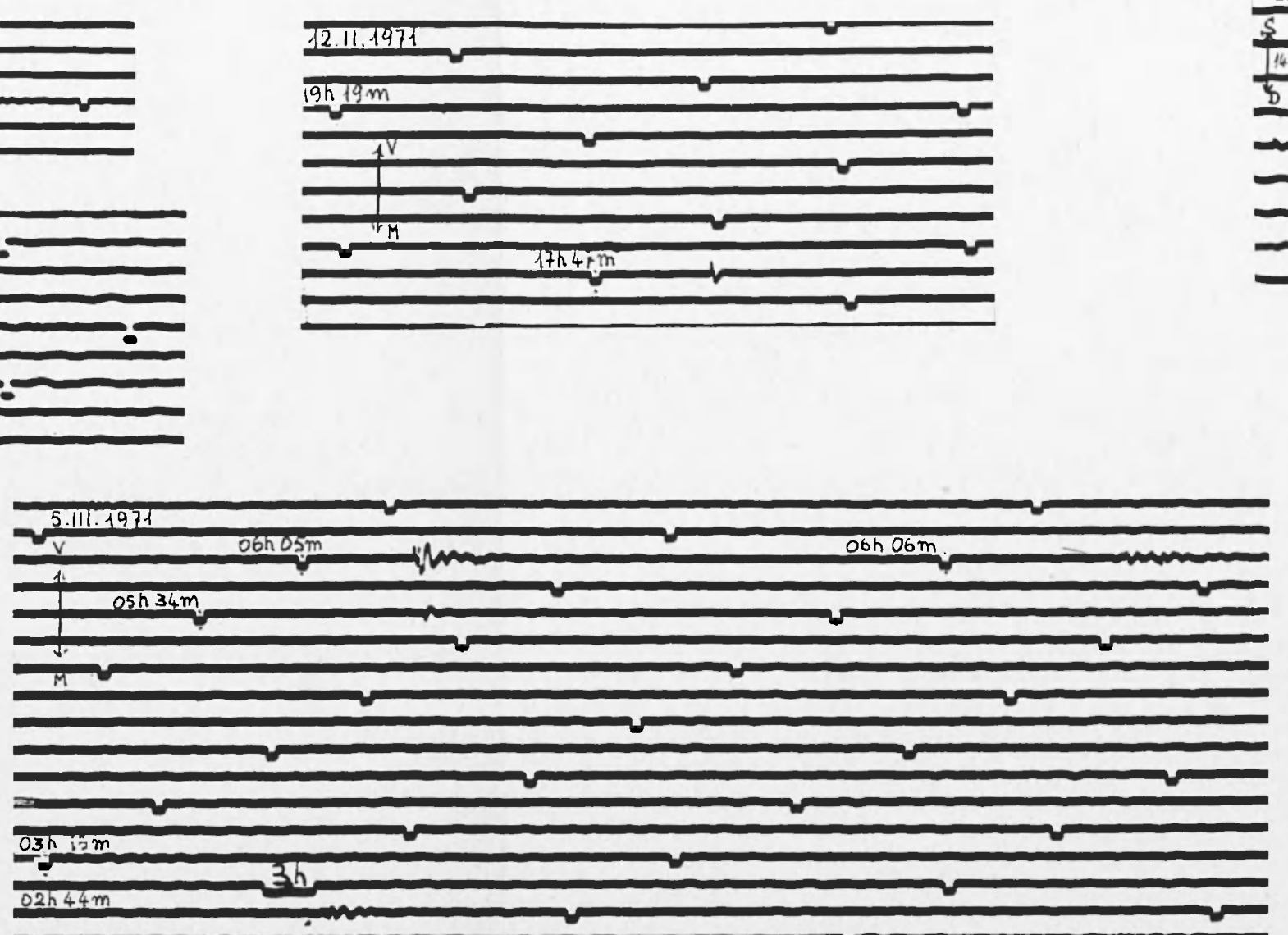
$\overline{\underline{23}}$ 12.11..19+1 $12 \mathrm{~h} 1 \mathrm{~m} / \mathrm{m}$ TM

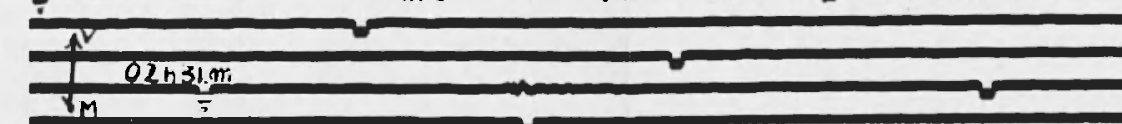

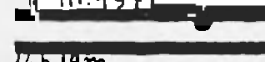

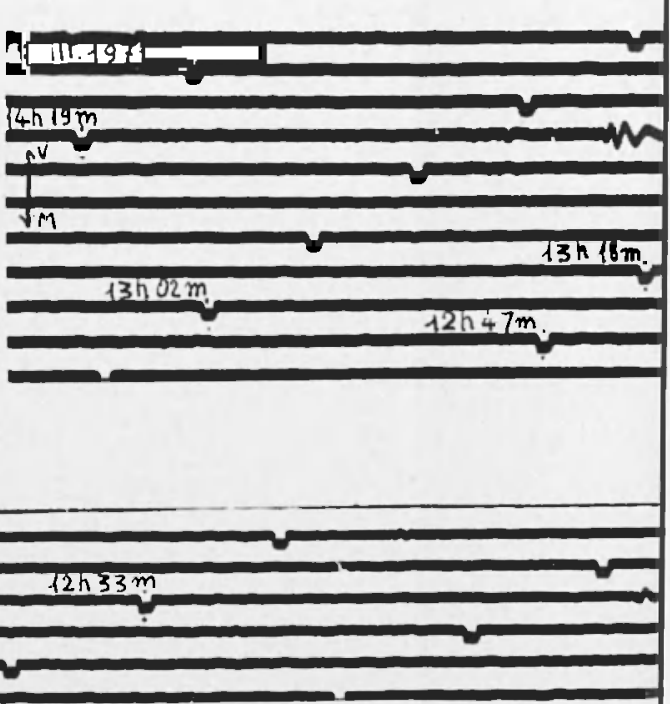

$\frac{26 . \times 11.1970-15 h 28 \mathrm{~m}}{15.15 g^{m}}$

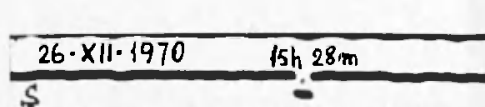
s $\frac{14.57}{5}-2-16=$  $=-$ $-\sqrt{-10.40}$

A2.1.1991 -

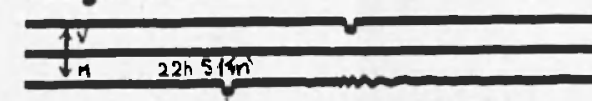
年 
COMPORTAMENTO DI UXA GRANDE DIGA SOTTO IL GELO SPINTO 513

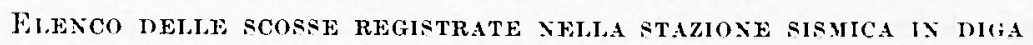
DAL 24-XII-1970 AL 27-XII-1970

\begin{tabular}{|c|c|c|c|c|c|c|c|c|c|}
\hline N. & Data & h & m & s & N. & Data & li & $\mathrm{m}$ & $s$ \\
\hline $\begin{array}{r}1 \\
\\
2 \\
3 \\
4 \\
5 \\
6 \\
7 \\
8 \\
9 \\
10 \\
11 \\
12 \\
13 \\
14 \\
15 \\
16 \\
17 \\
18 \\
19 \\
20 \\
21 \\
22 \\
23 \\
24 \\
25 \\
26 \\
27 \\
28 \\
29 \\
30 \\
31 \\
32 \\
33 \\
34 \\
35 \\
36 \\
37 \\
38 \\
39 \\
40 \\
41 \\
42 \\
43 \\
44 \\
45 \\
46 \\
47 \\
48 \\
49 \\
50\end{array}$ & $x^{2}$ & $\begin{array}{r}\left\{^{15}\right. \\
16 \\
17\end{array}$ & $\begin{array}{l}13 \\
34 \\
37 \\
40 \\
41 \\
48 \\
50 \\
27 \\
15 \\
28 \\
30 \\
40 \\
44 \\
48 \\
\\
50 \\
58 \\
08 \\
17 \\
31 \\
20 \\
26 \\
33 \\
38 \\
40 \\
44 \\
47 \\
51 \\
12 \\
25 \\
35 \\
48 \\
01 \\
46 \\
18 \\
28 \\
30 \\
49 \\
49 \\
49 \\
04 \\
15 \\
19 \\
22 \\
29 \\
34 \\
38 \\
44 \\
01 \\
10\end{array}$ & $\begin{array}{l}23.4 \\
24.9 \\
00 c a \\
17.8 \\
08.7 \\
23.5 \\
12.8 \\
53.0 \\
14.75 \\
14.1 \\
49.8 \\
51.3 \\
31.8 \\
28.0 \\
37.7 \\
49.7 \\
43.8 \\
20.8 \\
10.6 \\
43.2 \\
45.8 \\
07.2 \\
50.0 \\
30.5 \\
08.6 \\
52.1 \\
24.6 \\
37.7 \\
34.3 \\
38.4 \\
51.7 \\
13.8 \\
18.8 \\
13.4 \\
30.8 \\
29.3 \\
24.0 \\
21.1 \\
28.2 \\
36.2 \\
39.7 \\
50.5 \\
22.9 \\
04.4 \\
24.0 \\
06.7 \\
30.3 \\
03.1 \\
30.5 \\
37.8 \\
21.7\end{array}$ & $\begin{array}{r}51 \\
52 \\
53 \\
54 \\
55 \\
56 \\
57 \\
58 \\
59 \\
60 \\
61 \\
62 \\
63 \\
64 \\
65 \\
66 \\
67 \\
68 \\
69 \\
70 \\
71 \\
72 \\
73 \\
74 \\
75 \\
76 \\
77 \\
78 \\
79 \\
80 \\
81 \\
82 \\
83 \\
84 \\
85 \\
86 \\
87 \\
88 \\
89 \\
90 \\
91 \\
92 \\
93 \\
94 \\
95 \\
96 \\
97 \\
98 \\
99 \\
100 \\
101\end{array}$ & $25-X 1 I-1970$ & 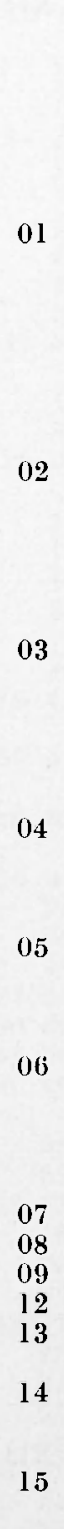 & $\begin{array}{l}17 \\
21 \\
29 \\
41 \\
43 \\
46 \\
56 \\
01 \\
02 \\
07 \\
19 \\
40 \\
51 \\
52 \\
54 \\
06 \\
10 \\
11 \\
13 \\
45 \\
52 \\
09 \\
14 \\
28 \\
28 \\
30 \\
40 \\
27 \\
44 \\
49 \\
54 \\
17 \\
26 \\
28 \\
47 \\
17 \\
33 \\
34 \\
46 \\
48 \\
42 \\
21 \\
58 \\
27 \\
53 \\
55 \\
03 \\
07 \\
45 \\
00 \\
06\end{array}$ & $\begin{array}{l}02.7 \\
44.3 \\
23.2 \\
41.3 \\
02.0 \\
30.5 \\
05.7 \\
24.5 \\
55.3 \\
52.3 \\
41.2 \\
15.3 \\
25.9 \\
01.8 \\
30.1 \\
02.4 \\
49.5 \\
06.4 \\
56.1 \\
58.1 \\
23.0 \\
34.2 \\
04.6 \\
46.4 \\
54.5 \\
29.0 \\
01.2 \\
18.9 \\
03.6 \\
34.0 \\
25.2 \\
21.7 \\
25.2 \\
16.4 \\
43.6 \\
10.9 \\
05.0 \\
13.6 \\
25.6 \\
01.9 \\
39.1 \\
41.8 \\
30.3 \\
44 \mathrm{ca} \\
56.5 \\
40 \mathrm{ca} \\
17 \mathrm{ca} \\
43 \mathrm{ca} \\
41 \mathrm{ca} \\
31.7 \\
28 \mathrm{ca}\end{array}$ \\
\hline
\end{tabular}


Serue elenco

\begin{tabular}{|c|c|c|c|c|c|c|c|c|c|}
\hline N. & 1)atat & h & $\mathrm{m}$ & $s$ & N. & Data & h & $\mathrm{III}$ & s \\
\hline 102 & $25 \cdot X I I-1970$ & & 06 & $43 \cdot: \mathrm{a}$ & 154 & $26 . X 1[-1970$ & 00 & 47 & $47 \mathrm{ca}$ \\
\hline 103 & & & 13 & 14.5 & 155 & & & 51 & 32.0 \\
\hline 104 & & & 14 & $04 c a$ & 156 & & & 59 & $29 c a$ \\
\hline 105 & & & 17 & $4 \mathrm{lca}$ & 157 & & ()1 & 27 & 3 lca \\
\hline 106 & & & 33 & 17.5 & 158 & & & 28 & $56 c a$ \\
\hline 107 & & & 58 & $38 \mathrm{ca}$ & 159 & & & 31 & 51.0 \\
\hline 108 & & 16 & 11 & $18 \mathrm{ca}$ & 160 & & () I & 34 & $47 \mathrm{ca}$ \\
\hline 109 & & & 16 & 3 lca & 161 & & & 46 & $08 \mathrm{ca}$ \\
\hline 110 & & & 24 & $15 \mathrm{ca}$ & 162 & & & 46 & 31.0 \\
\hline 111 & & & 25 & 40.5 & 163 & & 02 & 08 & 32.0 \\
\hline 112 & & & 34 & $55 \mathrm{ca}$ & 164 & & .02 & 29 & 08.3 \\
\hline 113 & & & 37 & 03.5 & 165 & & e & & 08.4 \\
\hline 114 & & & +5 & 47.5 & 166 & & & 41 & 41.0 \\
\hline 115 & & & +9 & 24.5 & 167 & & & 47 & $32 \mathrm{ca}$ \\
\hline 116 & & & 49 & 39.5 & 168 & & 03 & 00 & 21.5 \\
\hline 117 & & 17 & 06 & $55 \mathrm{ca}$ & 169 & & & 05 & 26.5 \\
\hline 118 & & & 14 & 39.5 & 170 & & & 21 & 56.0 \\
\hline 119 & & & 31 & $58 \mathrm{ca}$ & 171 & & & 22 & 13.0 \\
\hline 120 & & 18 & 35 & $36 \mathrm{ca}$ & 172 & & & 22 & 37.0 \\
\hline 121 & & 19 & 01 & 09.5 & 173 & & & 31 & 42.0 \\
\hline 122 & & & 07 & $06 \mathrm{ca}$ & 174 & & & 56 & $26 \mathrm{ca}$ \\
\hline 123 & & & 17 & 37.5 & 175 & & & 56 & $55 \mathrm{ca}$ \\
\hline 124 & & & 29 & $24 c a$ & 176 & & 04 & 14 & 09.0 \\
\hline 125 & & & 49 & 18.5 & 177 & & & 22 & $31 \cdot 0$ \\
\hline 126 & & & 59 & 21.5 & 178 & & & 30 & 57.5 \\
\hline 127 & & 20 & 07 & $20 \times a$ & 179 & & & 39 & 07.5 \\
\hline 128 & & & 30 & 58.5 & 180 & & & 47 & $45 \mathrm{ca}$ \\
\hline 129 & & 21 & 05 & 41.5 & 181 & & & 54 & $43 c a$ \\
\hline 130 & & $\{2]$ & 21 & 46.2 & 182 & & 05 & 48 & $13 c a$ \\
\hline 131 & & \{ & & +7.5 & 183 & & 06 & 02 & $04 \mathrm{ca}$ \\
\hline 132 & & & 22 & 38.0 & 184 & & & 07 & $31 \mathrm{ca}$ \\
\hline 133 & & $s 21$ & 33 & 17.6 & 185 & & & 25 & 57.0 \\
\hline 134 & & \{ & & 17.65 & 186 & & & 26 & $18 \mathrm{ca}$ \\
\hline 135 & & & 46 & 47.5 & 187 & & & 58 & 10.0 \\
\hline 136 & & & 52 & 28.0 & 188 & & 07 & 20 & 16.0 \\
\hline 137 & & 22 & 00 & 53.0 & 189 & & & 24 & 07.5 \\
\hline 138 & & & 02 & 30.0 & 190 & & & 31 & 33.5 \\
\hline 139 & & & 03 & 40.0 & 191 & & 08 & 21 & 47.0 \\
\hline 140 & & & 15 & $05 \mathrm{ca}$ & 192 & & & 55 & 13.0 \\
\hline 141 & & & 15 & $12 \mathrm{ca}$ & 193 & & s08 & 58 & 40.1 \\
\hline 142 & & & 28 & 25.0 & 194 & & & & 40.2 \\
\hline 143 & & s22 & 44 & 08.5 & 195 & & 09 & 19 & $36 \mathrm{ca}$ \\
\hline 144 & & & & 08.6 & 196 & & 11 & 28 & $07 \mathrm{ea}$ \\
\hline 145 & & & 54 & 57.5 & 197 & & 12 & 01 & $34 \mathrm{ca}$ \\
\hline 146 & & & 55 & $10 \mathrm{ca}$ & 198 & & & 09 & $07.5 \mathrm{ca}$ \\
\hline $1+7$ & & 23 & 06 & $42 \mathrm{ca}$ & 199 & & & 12 & $43 \mathrm{ca}$ \\
\hline 148 & & & 26 & 03.0 & 200 & & & 21 & $05 \mathrm{ca}$ \\
\hline 149 & & & 28 & 40.0 & 201 & & & 27 & $52 \mathrm{ca}$ \\
\hline 150 & & & 40 & 21.0 & 202 & & & 29 & $56 \mathrm{ca}$ \\
\hline 151 & $26-X 11-1970$ & 00 & 16 & 28.0 & 203 & & & 30 & $32 \mathrm{ca}$ \\
\hline 152 & & & 27 & 25.0 & 204 & & 12 & 33 & 13,7 \\
\hline 153 & & & 30 & $18 \mathrm{ca}$ & 205 & & & & 13.8 \\
\hline
\end{tabular}


Segne elenco

\begin{tabular}{|c|c|c|c|c|c|c|c|c|c|}
\hline S. & Mata & lı & III & $s$ & $\mathrm{~N}$ & Data & h & $m$ & $s$ \\
\hline $20 t i$ & $26 . \mathrm{XIJ}-1970$ & 12 & 34 & $43 c a$ & 258 & 26. XII-1970 & 16 & 50 & 10.7 \\
\hline 207 & & & 36 & $08 c a$ & 259 & & & & 10.8 \\
\hline 208 & & & 44 & $34 c a$ & 260 & & & 57 & 14.5 \\
\hline 209 & & & 46 & $47 \mathrm{ca}$ & 261 & & & 57 & 36.5 \\
\hline 210 & & & 51 & loca & 262 & & 17 & 02 & Ilea \\
\hline 211 & & 13 & 01 & $25 c a$ & 263 & & & 02 & $33 \mathrm{ca}$ \\
\hline 212 & & & 21 & $09 c a$ & 264 & & & 02 & $50 \mathrm{ca}$ \\
\hline 213 & & & 28 & $05 c a$ & 265 & & & 10 & 05.5 \\
\hline 214 & & & 33 & $34 c a$ & 266 & & & 14 & 38.5 \\
\hline 215 & & & 36 & $36 c a$ & 267 & & & 25 & $37 \mathrm{ea}$ \\
\hline 216 & & & 37 & $16 \mathrm{ca}$ & 268 & & & 30 & 47.0 \\
\hline 217 & & & 39 & $14 c a$ & 269 & & f 17 & 48 & 17.8 \\
\hline 218 & & & 39 & $53 \mathrm{ca}$ & 270 & & & & 18.0 \\
\hline 219 & & & 41 & $17 \mathrm{ca}$ & 271 & & & 51 & $40 \mathrm{ca}$ \\
\hline 220 & & & 42 & $20 \mathrm{ca}$ & 272 & & & 57 & 47.6 \\
\hline 221 & & & 43 & $08 \mathrm{ca}$ & 273 & & 18 & 06 & 00.8 \\
\hline 222 & & & 47 & $19 \mathrm{ca}$ & 274 & & & & 01.0 \\
\hline 223 & & & 48 & $09 c a$ & 275 & & & 10 & $48 \mathrm{ca}$ \\
\hline 224 & & & 48 & $34 c a$ & 276 & & & 13 & $28 \mathrm{ca}$ \\
\hline 225 & & & 51 & $21 \mathrm{ca}$ & 277 & & & 18 & $38 \mathrm{ca}$ \\
\hline 226 & & 14 & 09 & $060 a$ & 278 & & & 35 & $27 \mathrm{ca}$ \\
\hline 227 & & & 26 & 07ca & 279 & & & 15 & $40 \mathrm{ca}$ \\
\hline 228 & & & 31 & $34 c a$ & 280 & & & 40 & $48 \mathrm{ca}$ \\
\hline 229 & & & 37 & $09 \mathrm{ca}$ & 281 & & 19 & 44 & 40.0 \\
\hline 230 & & & 43 & $12 \mathrm{ca}$ & 282 & & & & 40.05 \\
\hline 231 & & & 50 & $55 \mathrm{ca}$ & 283 & & 19 & 47 & 40.7 \\
\hline 232 & & & 57 & $12 c a$ & 284 & & & & 40.8 \\
\hline 233 & & & 57 & $14.5 \mathrm{ca}$ & 285 & & 19 & 51 & 10.3 \\
\hline 234 & & 15 & 03 & $57 \mathrm{ca}$ & 286 & & & & 10.35 \\
\hline 235 & & $\{15$ & 08 & 03.0 & 287 & & 20 & 01 & $25 \mathrm{ca}$ \\
\hline 236 & & & & 03.5 & 288 & & & 25 & $35 \mathrm{ca}$ \\
\hline 237 & & 615 & 12 & 40.5 & 289 & & & 38 & 41.6 \\
\hline 238 & & $\lambda$ & & 40.55 & 290 & & & 48 & 57.6 \\
\hline 239 & & & 14 & $55 \mathrm{ca}$ & 291 & & 21 & 54 & 21.5 \\
\hline 240 & & & 24 & 06,2 & 292 & $27-X I 1-1970$ & 00 & 39 & $05 \mathrm{ca}$ \\
\hline 241 & & 16 & 02 & 03.1 & 293 & & 01 & 26 & $45 \mathrm{ca}$ \\
\hline 242 & & & 05 & $28 \mathrm{ca}$ & 294 & & 01 & 27 & 23,1 \\
\hline 243 & & & 08 & 56.1 & 295 & & \{ & & 25.6 \\
\hline 244 & & & 09 & 03.4 & 296 & & & 48 & $42 \mathrm{ca}$ \\
\hline 245 & & & 13 & 38.1 & 297 & & & 51 & $150 a$ \\
\hline 246 & & 516 & 13 & 45.8 & 298 & & 02 & 13 & $57 \mathrm{ca}$ \\
\hline 247 & & \{ & & 45.85 & 299 & & & 16 & $49 \mathrm{ca}$ \\
\hline 248 & & & 17 & $33 \mathrm{ca}$ & 300 & & & 20 & $04 c a$ \\
\hline 249 & & & 17 & $39 \mathrm{ca}$ & 301 & & & 38 & $24 \mathrm{ca}$ \\
\hline 250 & & & 20 & $27 \mathrm{ca}$ & 302 & & 03 & 02 & $05 \mathrm{ca}$ \\
\hline 251 & & & 25 & $08 c a$ & 303 & & & 26 & 45.0 \\
\hline 252 & & & 28 & 3 lea & 304 & & & 32 & 04.6 \\
\hline 253 & & 16 & 31 & 11.6 & 305 & & & 33 & 27.5 \\
\hline 254 & & & & 11.8 & 306 & & & 52 & $00 \mathrm{ca}$ \\
\hline 255 & & 616 & 38 & 09.1 & 307 & & 04 & 01 & $46 \mathrm{ca}$ \\
\hline 256 & & $i$ & & 09.15 & 308 & & & 11 & 31.6 \\
\hline 257 & & & 49 & 07ea & 309 & & & 33 & $22 \mathrm{ca}$ \\
\hline
\end{tabular}


Segue elenco

\begin{tabular}{|c|c|c|c|c|c|c|c|c|c|}
\hline N. & Jata & h & m & $s$ & $N$ & Dat a & 11 & m & s \\
\hline $\begin{array}{l}310 \\
3 I I \\
3 I 2 \\
3 I 3 \\
3 I 4 \\
3 I 5 \\
3 I 6 \\
317 \\
318 \\
319 \\
320 \\
321 \\
322 \\
323 \\
324 \\
325 \\
326 \\
327 \\
328 \\
329 \\
330 \\
331\end{array}$ & 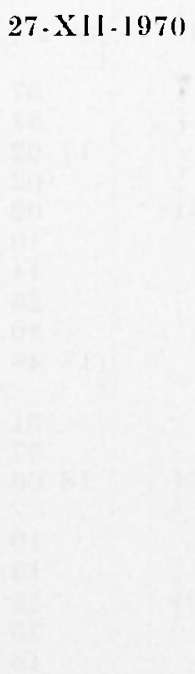 & $\begin{array}{l}05) \\
06\end{array}$ & $\begin{array}{l}38 \\
48 \\
05 \\
22 \\
32 \\
14 \\
28 \\
31 \\
32 \\
50 \\
56 \\
01 \\
06 \\
11 \\
18 \\
23 \\
29 \\
31 \\
05 \\
07 \\
08 \\
40\end{array}$ & $\begin{array}{l}04 \mathrm{ca} \\
43.5 \\
10.5 \\
55 \mathrm{ca} \\
56 \mathrm{ca} \\
13 \mathrm{ca} \\
18 \mathrm{ca} \\
08 \mathrm{ca} \\
52 \mathrm{ca} \\
45 \mathrm{ca} \\
07 \mathrm{ca} \\
44 \mathrm{ca} \\
58 \mathrm{ca} \\
06.5 \\
53 \mathrm{ca} \\
17.5 \\
38.5 \\
15 \mathrm{ca} \\
29.5 \\
16 \mathrm{a} a \\
43 \mathrm{ca} \\
25 \mathrm{ca}\end{array}$ & $\begin{array}{l}332 \\
333 \\
334 \\
335 \\
336 \\
337 \\
338 \\
339 \\
340 \\
341 \\
342 \\
343 \\
344 \\
345 \\
346 \\
347 \\
348 \\
349 \\
350 \\
351 \\
352 \\
353\end{array}$ & $27-\mathrm{X} I 1.1970$ & $\begin{array}{l}08 \\
09 \\
\\
10 \\
11 \\
12\end{array}$ & $\begin{array}{l}52 \\
07 \\
24 \\
36 \\
00 \\
53 \\
10 \\
23 \\
35 \\
58 \\
00 \\
17 \\
17 \\
27 \\
52 \\
19 \\
57 \\
08 \\
30 \\
51 \\
24 \\
10\end{array}$ & $\begin{array}{l}22.5 \\
39 \mathrm{ca} \\
13.5 \\
39.5 \\
21.5 \\
22 \mathrm{ca} \\
17.5 \\
22 \mathrm{ca} \\
43 \mathrm{ca} \\
13.5 \\
12.5 \\
28 \mathrm{ca} \\
34.5 \\
19 \mathrm{ca} \\
20 \mathrm{ca} \\
34 \mathrm{ca} \\
19.5 \\
44.5 \\
29 \mathrm{ca} \\
03.5 \\
49 \mathrm{ca} \\
43 \mathrm{ca}\end{array}$ \\
\hline
\end{tabular}

4. - A prescindere dalla base dello sbarramento, che ì particolarmente (se non esclusivamente) sensibile alle variazioni dell'invaso, il ciclo delle flessioni di una grande diga - a parità di altre condizioni - ̀̀ quindi regolato dall'andamento delle stagioni e, in modo particolare, dal comportamento anomalo dell'acqua, nei confronti delle variazioni della temperatura.

Sostanzialmente, può essere cosi riassunto. Consideriamo una diga (nel caso specifico, la diga di Pieve di Cadore) con l'annesso bacino idrico a livello normale. Ad una certa data dei primi mesi dell'anno (che a seconda dell'andamento stagionale, può coincidere col mese di Febbraio o di Marzo), l'acqua e l'aria vengono a trovarsi ad una temperatura media pressoché uguale. A partire da questa data, procedendo verso la primavera, l'aria comincia a riscaldarsi più dell'acqua. ì noto, infatti, che l'acqua ha il calore specifico più grande di tutte le sostanze conosciute; per cui, il suo riscaldamento avviene con molta lentezza, anche nei confronti del sistema roccioso, sopportante la diga. Pertanto, l'aumento del divario fra la temperatura media dell'aria 

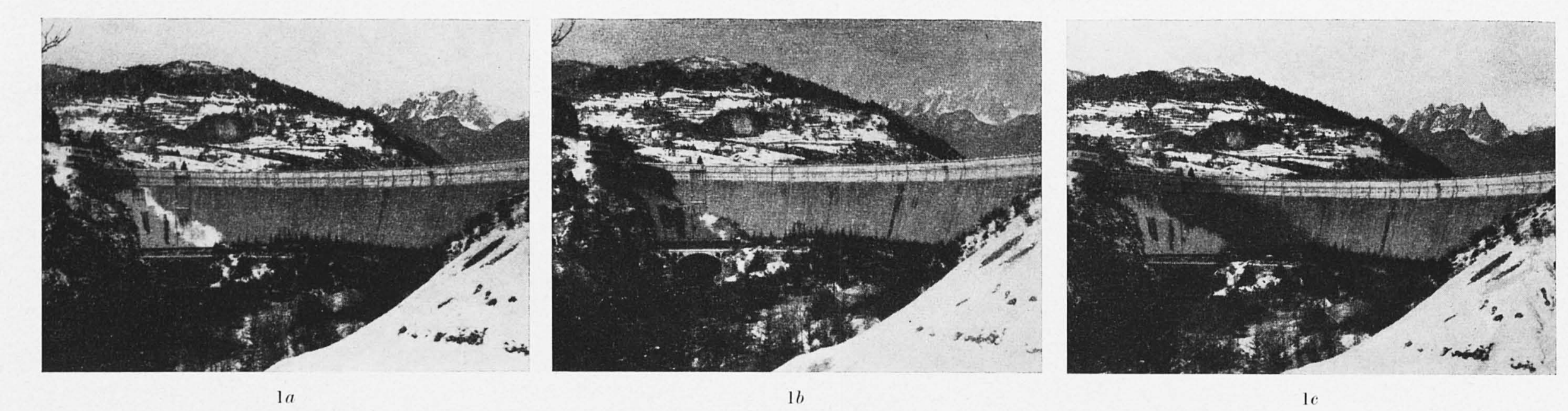

Foto $1 a)-1 b)-1 c)-1 d$ ): $10 . \mathrm{I} .1969$ alle $11 \mathrm{~h} 40 \mathrm{~m}, 12 \mathrm{~h}, 14 \mathrm{~h}, 15 \mathrm{~h}$.
Photo $1 a)-1 b)-1 c)-1 d$ ) : 10.I.1969. Insolation at $1 \mathrm{hh} 40 \mathrm{~m}, 12 \mathrm{~h}, 14 \mathrm{~h}, 15 \mathrm{~h}$.
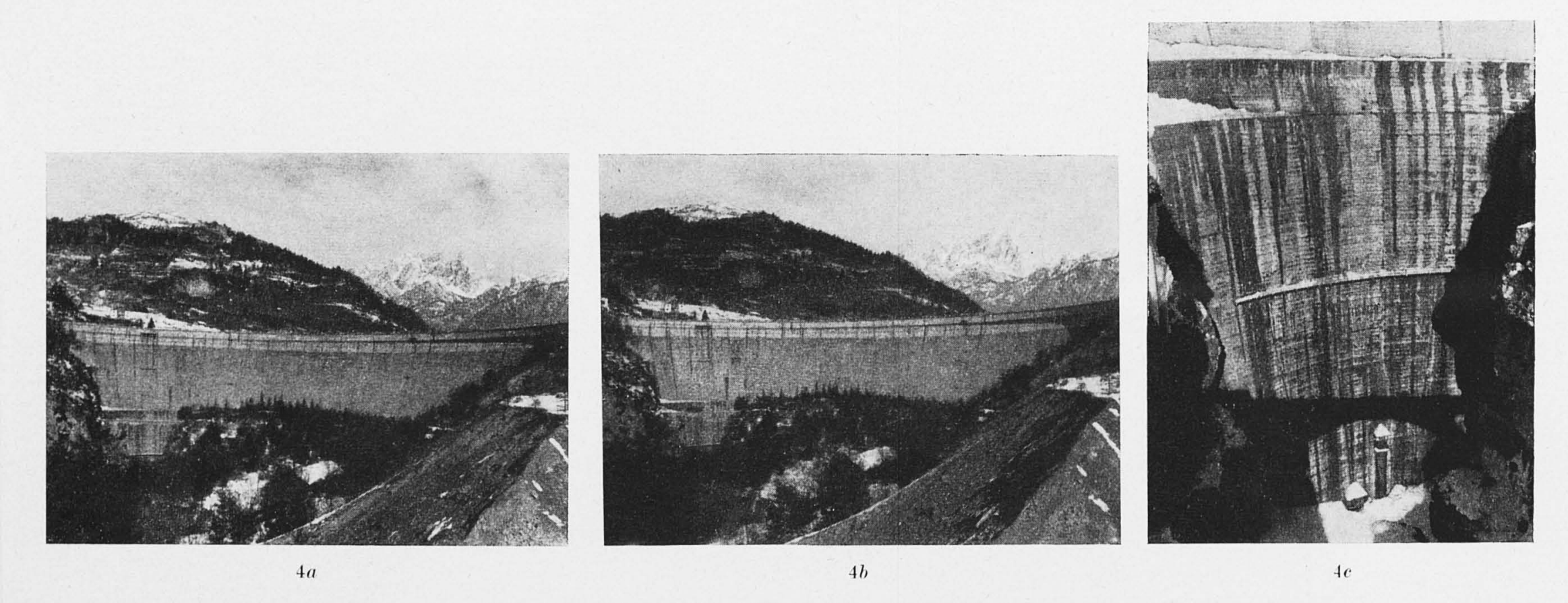

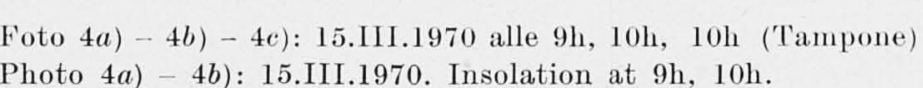

$\begin{array}{lll}\text { Photo } 4 c & \text { : Insolation at the } 10 \mathrm{~h} \text { of the same day on } \\ \text { Phot } & \end{array}$

the right side of the dan
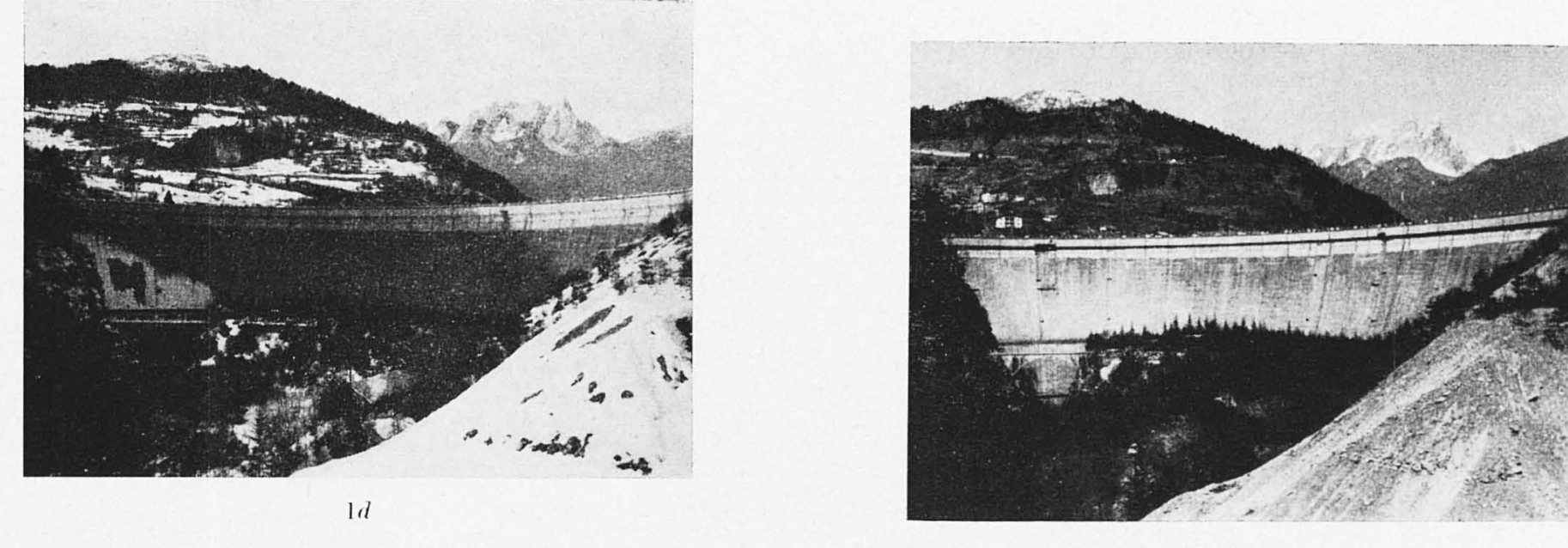

Foto 2: 9.IV.1969 alle 12h.
Photo 2: 9.IV.1969. Insolation at noon

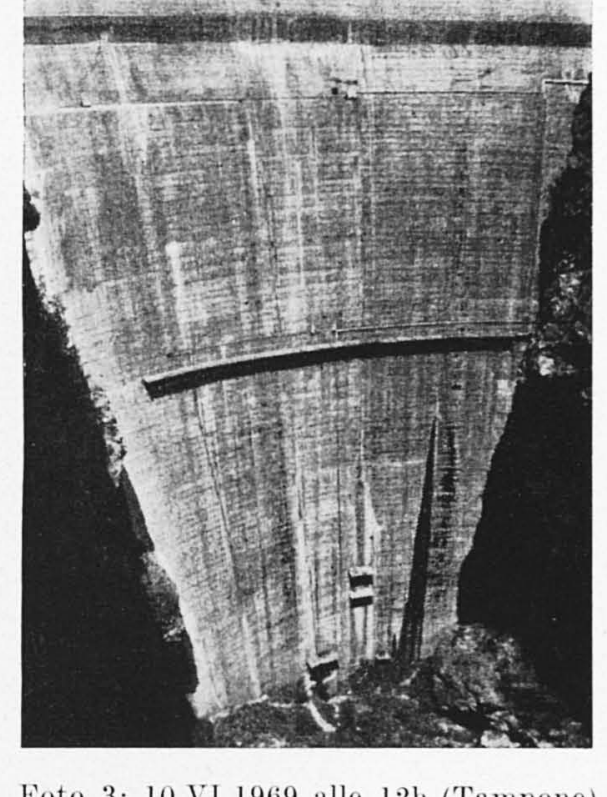

Foto 3: 10.VI.1969 alle $12 \mathrm{~h}$ (Tampone)
Photo $3: 10$ VI 1969 . Inolation on the to $3: 10.71 .1969$. Insolation on the
nat noon.
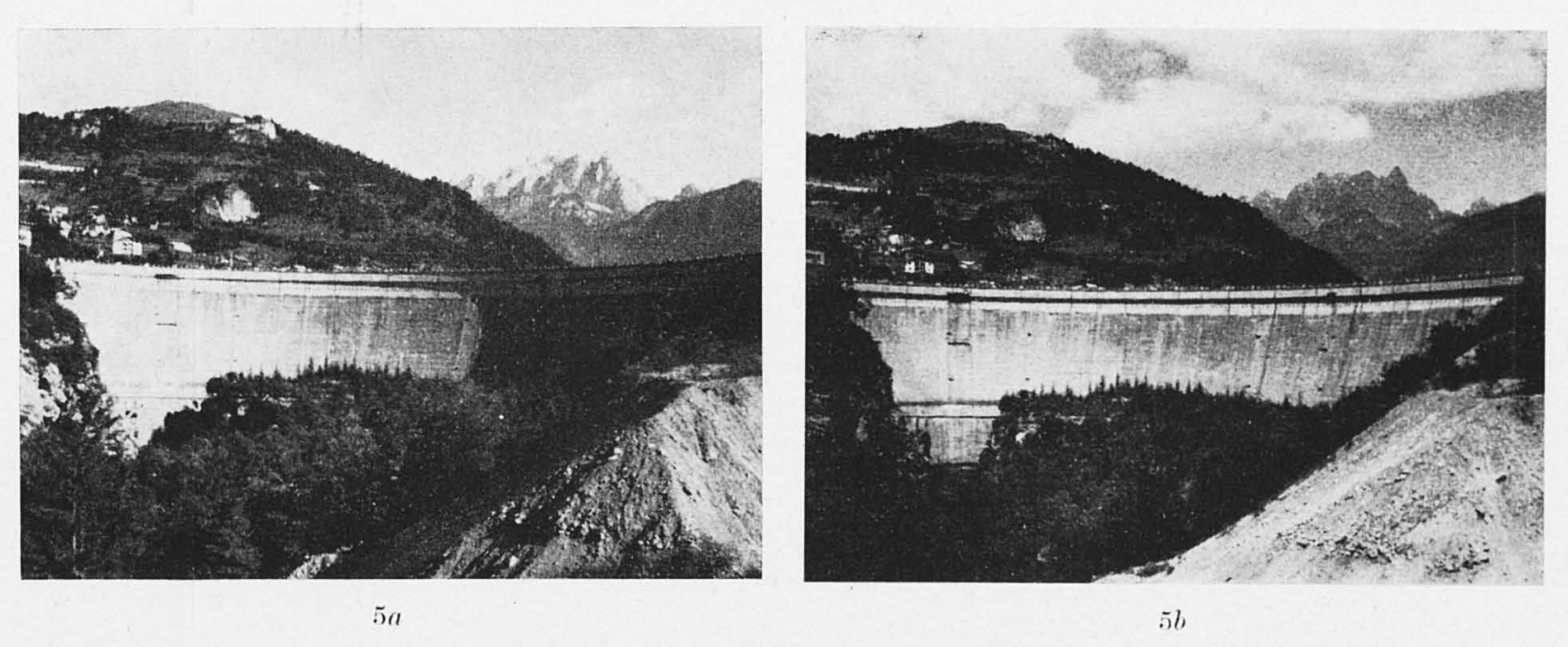

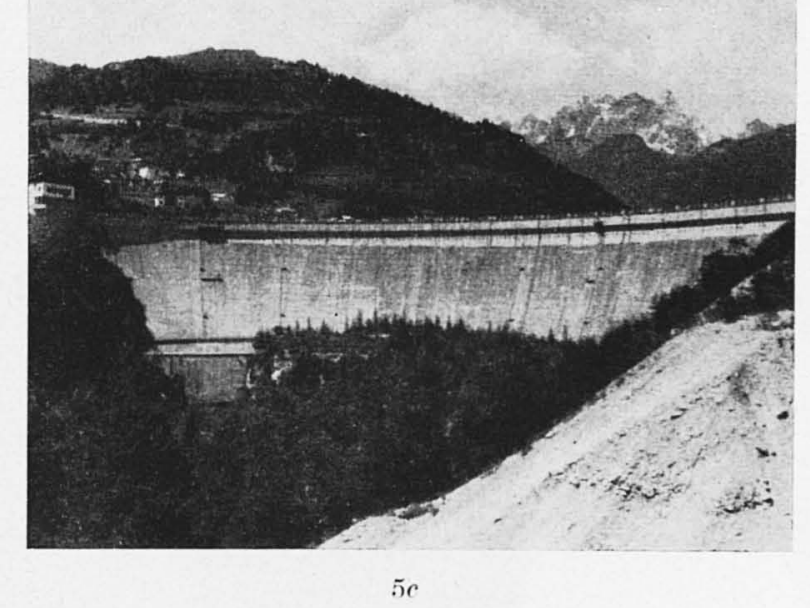


e quella dell'acqua, fa sì che la parete a valle della diga si riscalldi in maggior misura di quella a monte, immersa nel liquido, con la conseguenza di una flessione dello sbarramento verso monte, flessione che continua nei mesi successivi, mediamente fino a tutto Settembre. A questa lenta flessione verso monte, si sovrappone inoltre l'oncla diurna, dovuta all'andamento dell'insolazione nelle 24 ore.

In tutti questi mesi l'acqua non ha fatto che immagazzinare calore, portanclosi a temperature che, nello strato superficiale, possono raggiungere i $20^{\circ} \mathrm{C}$. Come lentamente ì avvenuto l'accumulo di calore, altrettanto lentamente l'acqua lo restituisce all'intorno. Cosi, quando la temperatura esterna comincia a diminuire, in breve volger di tempo la temperatura media dell'aria scende ad un livello inferiore di quello dell'acqua del lago. $\grave{E}$ a questo punto che la situazione termica delle pareti della diga, si inverte: la parete a nord viene infatti a trovarsi a temperatura maggiore di quella a sur. Si inverte quindi anche il fenomeno di flessione, che d'ora in avanti si verifica verso valle. Con l'ulteriore diminuzione della temperatura verso lo zero, nell'acqua del bacino idrico inizia un ininterrotto rimescolamento: l'acqua di superficie, raffredclandosi, si appesantisce e scendle di qualche metro, per essere sostituita da acqua più calda di quote inferiori. Se le condizioni esterne lo consentono, questo fenomeno può continuare fino al totale rimescolamento del lago, che verrà arl avere sul fondo temperature dell'ordine di 4 ' $\mathrm{C}$, alle quali - com'è noto - l'acqua ha la massima densiti, e quindi il massimo peso specifico. Questa situazione ultima, presuppone allesterno temperature nettamente sotto lo zero: di qui il formarsi di uno strato di ghiaccio alla superficie del lago, il continuare della flessione di tutta la diga verso valle - come è stato osservato al principio -; e, nel caso di gelo spinto, il verificarsi del fenomeno accennato di una parte della diga - quella di base - flettente verso monte, mentre la parte a quota superiore è sempre tesa in senso opposto. È in questa fase che il contrasto fra roccia e diga puó tradursi superato il limite di fermezza del mezzo - in centinaia di microscosse.

Naturalmente, quello esposto è l'andamento del fenomeno nei suoi aspetti più semplici, quando le grandezze in ginoco variano in uno stesso senso con uniformità. In effetti, si verificano complicazioni, legate a ritorni di stagione, ai quali è particolarmente sensibile la temperatura dell'aria; di qui, la pronta risposta dello sbarramento, sotto forma di inversioni di flessione, più o meno accentuati, più o meno lunghi, più o meno regolari, a seconda dell'andamento della 
causa. Senza accennare, naturalmente, alla concomitante azione sulla valle nel suo complesso, nelle sue parti solide: la quale tende a restringersi, nella stagione calla, e arl allargarsi in quella fredrla. Per tacere, infine, di tutti i contrasti, conseguenti alle variazioni, più o meno marcate, del livello dell'invaso.

\section{BIBLIOGRAFIA}

(1) CALOI P., 1953. - Osservazioni sismiche e clinografiche presso grandi dighe di sbarramento. "Annali di Geofisica ", VI, 3.

$\left({ }^{2}\right)$ Calor I', 1962. - La Geofisica e la grandi dighe. "L'Energia Elettrica", XXXIX, 1 .

$\left.{ }^{3}\right)$ Caior P., 1962. - Aspetti della dinamica di rocce, calcestruzzo ed acque. "Annali di Geofisica ", XV, 2-3. (Contiene un'ampia bibliografia su precedenti lavori (ell' $\mathrm{A}$. sull'argomento).

(1) Calor P., Spadea M. C., 1966. - Principali risullati conseguiti durante l'osservazione geodinamica, opportunamente estesa nel tempo, di grandi diglue di sbarramento, e loro giustificazioni teoriche. "Annali di Geofisica ", XIX, 3.

$\left(^{5}\right)$ Catoi P., Spadea M. C., 1969. - Sulla risposta elastica delle dighe e sulla sua variazione del tempo. "Annali di Geofisica", XXII, 1.

$\left(^{\circ}\right)$ Fukuo Y., 1966. - On the Rheological Behavior of Frozen Soil (Part I). "Bull. Disaster Prevention Res. Inst. ", 15, part 3.

(7) Scineidegger E. A., 1970. - Theoretical Geomorphology. Seronda Edizione riveduta. Springer-Verlag. Berlin, pp. 375-382. 